\title{
Impresión 3D como herramienta docente en asignaturas de Resistencia de Materiales*
}

\author{
Andrés Díaz ${ }^{1}$, Isidoro Iván Cuesta ${ }^{1}$ y Jesús Manuel Alegre ${ }^{1}$ \\ ${ }^{1}$ Universidad de Burgos, Escuela Politécnica Superior, Avda. Cantabria s/n. 09006
}

\begin{abstract}
$3 D$ printing is an increasingly popular technology that is being proposed from different approaches as a teaching tool. Besides its application in the production of visual and representative objects, 3D printing is proposed here for the manufacture of specimens that are then tested with the aim of characterising mechanical properties of materials within the teaching framework of Solid Mechanics courses. This methodology is able to engange sutdents in the desing, printing and test of the specimens. As a result, the process of material characterisation in the lab is faster and might be completely integrated as a supporting teaching tool of different testing procedures related to the Structural Integrity of materials and components.
\end{abstract}

Keywords: 3D printing, Teaching tools, Mechanics of Materials

\begin{abstract}
Resumen
La Impresión $3 D$ es una tecnología en auge que está siendo propuesta desde diversos enfoques como una herramienta docente. Además de su utilidad como técnica de creación de objetos visuales y representativos, aqui se propone la fabricación de probetas típicas para la caracterización de las propiedades mecánicas de distintos materiales dentro del contexto docente de la Resistencia de Materiales. La metodología desarrollada hace partícipe al alumno en el proceso de diseño, impresión y ensayo de las probetas. De este modo, el proceso de caracterización se agiliza en el laboratorio permitiendo su completa incorporación como apoyo a la docencia de diversos ensayos relacionados con la Integridad Estructural de materiales y componentes.
\end{abstract}

Keywords: Impresión 3D, Herramientas docentes, Resistencia de Materiales

\footnotetext{
*Proyecto llevado a cabo por el Grupo HERIN (Herramientas Ingenieriles para la Innovación y Mejora Docente)
} 


\section{Introducción}

La asociación de conceptos abstractos con su correspondiente materialización tangible es un aspecto clave en el proceso de aprendizaje. En este sentido, la Impresión 3D está siendo ampliamente aceptada en disciplinas donde los modelos son difícilmente obtenibles y reproducibles. Cabe destacar los modelos anatómicos en enseñanzas de medicina (Vaccarezza y Papa 2015; Kong y col. 2016) y los modelos atómicos o moleculares en física y ciencia de materiales (Rodenbough, Vanti y Chan 2015). También en aquellas materias relacionadas directamente con la geometría y las técnicas de representación (Huleihil 2017).

En enseñanzas tecnológicas la incorporación de la Impresión 3D tiene un doble valor: mientras se desarrollan objetos de representación útiles para la asimilación de contenidos e ideas, al mismo tiempo se está familiarizando al alumno con una técnica novedosa con potencial impacto en muchas ramas científico-técnicas e industriales. Parece evidente que la formación de alumnos en etapas universitarias de grado y postrgrado en tecnologías de Impresión 3D aumentará sus habilidades profesionales y sus perspectivas de empleabilidad.

A pesar de la proyección de la tecnología de Fabricación Aditiva en la industria se corre el riesgo, como en todo boom tecnológico, de encontrarnos en el pico de sobreexpectación del conocido como Hype Cycle o Ciclo de Gartner (Kietzmann, Pitt y Berthon 2015). Si la docencia puede ayudar a confirmar la Impresión 3D como una tecnología madura y sólida con la creación de nuevos profesionales capaces o si en cambio contribuirá a una posible burbuja es un debate que está fuera del alcance de este trabajo. No obstante, aquí se proponen enfoques metodológicos prácticos en los que el objetivo de aprendizaje es sustancialmente clásico, conceptos de mecánica y resistencia de materiales, aunque una tecnología innovadora se establezca como herramienta conductora de la docencia.

El objetivo principal de este trabajo es revisar el marco teórico de la Impresión 3D como herramienta docente y destacar las ventajas de su implementación en asignaturas de Resistencia de Materiales en carreras técnicas. El enfoque es innovador en el sentido en que el objeto impreso no solo es un apoyo sensorial en el proceso de aprendizaje, como suele se habitual en la docencia a través de la impresión 3D, sino que se constituye además como objeto de análisis resistente.

El presente trabajo se estructura de la siguiente manera: se parte de un análisis teórico de la utilidad de la Impresión 3D en el aprendizaje desde el punto de vista cognoscitivo para, a continuación, exponer las características principales de esta tecnología dentro de un marco docente. Se hace especial hincapié en su idoneidad en contextos de aprendizaje activo dentro de un proceso que abarque desde la etapa de concepción a la aplicación final. Finalmente, se detalla la metodología de aula-laboratorio propuesta para la aplicación de las ideas previamente expuestas en el enseñanza de Resistencia de Materiales e Integridad Estructural. No se describe aquí una experiencia práctica concreta con un guión definido ni se limita la aplicación a una asignatura o grado determinado. Aunque se hable de forma genérica de Impresión 3D o Fabricación Aditiva, la técnica implementada, así como en la práctica totalidad de experiencias docentes, es la denominada como Fused Deposition Modelling. 


\section{Modelos de realidad}

Como se ha mencionado en la introducción, el manejo de conceptos abstractos durante el aprendizaje puede y debe apoyarse en un pensamiento más concreto. Tres conceptos se discuten a continuación alrededor de la relación abstracción-realidad: ilustración, analogía y generalización. Quizá la forma más sencilla de transmitir conocimiento complejo sea a través de ejemplos concretos. La ilustración puede llevarse a cabo mediante canales orales, escritos o visuales. Por otro lado, la analogía ahorra ese esfuerzo de abstracción tratando de aprovechar conocimientos previos (Gentner y Holyoak 1997). A pesar de la utilidad de la ilustración y la analogía, debe tenerse siempre presente que, especialmente en enseñanzas técnicas, la generalización o ley constituye una de las metas básicas del método científico.

Aunque la mayor parte de la aplicación de la Impresión 3D se centra en el concepto de ilustración, no deben descuidarse el papel fundamental de la analogía y la deducción de leyes en el aprendizaje. La ilustración mediante la tecnología 3D se integra en un escenario de aprendizaje tangible donde se introducen lo que aquí llamamos modelos de realidad. Esta denominación simplemente trata de abarcar todas aquellas estrategias de representación de realidades complejas en un escenario adaptado. Los ejemplos más destacados son los modelos de realidad virtual o Realidad Aumentada, con un gran interés por parte de educadores a al hora de facilitar una experiencia realista que ayude al proceso de aprendizaje (Dunleavy y Dede 2014). La idea es sencilla: ilustraciones o experiencias en 3D mejoran el aprendizaje respecto a representaciones 2D (Huk 2006), entornos dinámicos respecto a estáticos (Meij y Jong 2006), entornos realistas respecto a simbólicos (Lateef 2010), etcétera.

A pesar del aparente consenso sobre el papel de la visualización en el aprendizaje, cabe preguntarse hasta qué punto el realismo de los materiales visuales, es decir, el parecido entre representación y realidad influye en los procesos cognitivos. Es interesante el enfoque que Winn 1982 hace sobre la relación entre percepción y asimilación, concluyendo que "la información se representa internamente como esquemas, pudiendo contener o no representaciones realistas de la realidad". Si bien la impresión 3D actúa como estrategia instructiva visual, no debe ser sustitutivo de otras estrategias de aprendizaje más abstractas. De nuevo, el equilibrio entre la ilustración particular, la analogía y la ley general debe ser analizado; el debate entre lo concreto y lo abstracto en los procesos cognitivos es fundamental.

Hay que destacar que, aunque fuera del objetivo de este trabajo donde solo se tratan los objetos tangibles en su función exploratoria y como modelos de realidad, la Impresión 3D abre las puertas a nuevas herramientas en las que la función de ilustración es sustituida por una de expresión y creatividad (Marshall, Price y Rogers 2003).

\section{Impresión 3D en la docencia}

Ford y Minshall 2018, distinguen cinco usos de la impresión 3D en la docencia:

1. Enseñar Impresión 3D a estudiantes.

2. Enseñar Impresión 3D a docentes.

3. Enseñar habilidades de diseño y creatividad. 
4. Producir artefactos que apoyen la docencia.

5. Crear tecnologías de apoyo.

Excepto la quinta categoría, necesaria solamente en Educación Especial o en aquellos casos en que haya problemas de funcionalidad en uno o varios alumnos, las otras cuatro variantes se presentan de manera sinérgica en la mayoría de escenarios docentes, incluido el expuesto en este trabajo.

La formación de los docentes en Impresión 3D es un requisito al que no se le ha prestado la suficiente atención a la hora de proponer la introducción de este tipo de tecnologías en las diferentes etapas educativas. A pesar de que hay muchos recursos y proyectos colaborativos disponibles, es inmenso el esfuerzo y tiempo que debe dedicarse para poder manejar esta tecnología y después incorporarla en los ajustados planes docentes. Otra dificultad añadida es el coste de los equipos y materiales que, si bien está reduciéndose con la extensión de las Impresoras 3D de escritorio, puede suponer una barrera para su uso en los centros educativos.

\section{Aprendizaje activo}

Numerosos conceptos de innovación docente podrían aplicarse mediante la Impresión 3D en el aula: Aprendizaje Basado en Proyectos (ABP) (Lacey 2010), Do-It-Yourself (De Jong y Bruijn 2013), gamificación (Pérez y col. 2015), pensamiento visual y espacial (Katsioloudis y Jones 2015), etcétera. Sin entrar en detalle, se destaca el papel del aprendizaje activo y la participación e implicación por parte del alumno en la construcción de los recursos docentes. En carreras técnicas, el estudiante puede controlar el proceso de diseño e impresión 3D, ya que cuenta con nociones de dibujo CAD, una vez se le familiariza con el manejo de archivos .stl y los software de impresión por capas (slicing softwares). Hay que destacar que la Impresión 3D es una tecnología que ha surgido con un espíritu de acceso abierto y recursos compartidos por lo que existen multitud de softwares de acceso abierto (Cura o Slic3r) y repositorios.

Dando un paso más allá, algunos proyectos han hecho partícipe al alumno en el ensamblaje de la propia Impresora 3D (Martin, Bowden y Merrill 2014). Esta actividad es especialmente adecuada para Ingenierías Mecánicas, Electrónicas o Informáticas, donde el desarrollo de una Impresora 3D puede apoyar la transmisión de ciertos conocimientos técnicos.

En la práctica propuesta en la siguiente sección se han seguido los preceptos que incluye el marco CDIO (Conceive - Design - Implement - Operate). La iniciativa CDIO fue desarrollada originariamente en el Massachusetts Institute of Technology (MIT) en la década de los 90 (Villegas 2011). Realmente cualquier aprendizaje activo y basado en proyectos debería ir encaminado a cumplir las cuatro fases descritas a continuación de manera particular para la Impresión 3D en la docencia:

- Conceive. En la docencia de la Impresión 3D se corre el riesgo de minimizar los tiempos dedicados a la concepción, es decir, a la identificación de problemas y posibles soluciones. Tanto la implicación del alumno como el establecimiento de los esquemas conceptuales necesarios para el posterior aprendizaje activo son construidos durante esta etapa inicial. En el caso concreto de la aplicación a problemas relacionados con la Resistencia de Materiales, es fundamental la 
capacidad predictiva, es decir, qué el alumno sea capaz de adelantarse a los resultados en cierto modo teniendo en cuenta los conceptos teóricos previamente tratados.

- Design. El diseño de piezas a través de programas CAD y su posterior tratamiento como archivo .stl es clave en la docencia de la Impresión 3D. Aunque en ciertas disciplinas relacionadas con diseño gráfico, dibujo técnico o técnicas de representación puedan hacer más hincapié en este proceso, en otros casos se podrá recurrir a librerías de ficheros o acelerar el diseño facilitando al alumno algunos archivos. En cualquier caso, habrá ciertos parámetros que deban ser explicados y discutidos en relación a las características del sólido generado para su posterior análisis resistente; de este modo, influirán críticamente el tipo de material, el porcentaje de relleno, el tamaño y distribución de las capas, etcétera.

- Implement. En este caso, se trata del proceso de Impresión o incluso del montaje y calibración de la impresora en aquellos proyectos en los que los alumnos sean partícipes en dicho proceso.

- Operate. La etapa final de ejecución en un entorno real en la que se basa el enfoque CDIO aquí se traduce como un ensayo en laboratorio, de la manera que se realizaría en cualquier investigación real de materiales tanto en el mundo académico como en la industria. Esta última fase se detalla en la siguiente sección donde se explican los conceptos relacionados con la Resistencia de Materiales y los tipos de ensayo que pueden incluirse.

Resumiendo, el proceso CDIO trasladado a la metodología propuesta será: concepción del problema, diseño de piezas, impresión en 3D y ensayo en laboratorio.

\section{Ensayo de probetas impresas en 3D}

Esta sección trata de servir de guía para una metodología práctica que permita al docente diseñar una serie de sesiones encaminadas a la enseñanza de determinados conceptos de la Resistencia de Materiales como disciplina clásica con la tecnología de Impresión 3D como herramienta auxiliar y conductora del proyecto. Siendo las secciones anteriores de carácter general sobre el papel docente de la Impresión 3D, a continuación se emplearán conceptos técnicos con los que el lector general puede no estar familiarizado.

La Impresión 3D no deja de ser un proceso de fabricación, es decir, una alternativa otros procesos como la fundición, el moldeo o la forja. En todos ellos, una materia o conjunto de materias primas se transforma para tener una forma y unas propiedades determinadas. En el caso de la Impresión 3D, en concreto para la técnica de Fused Deposition Modelling, el material input es un filamento de un polímero que se funde a alta temperatura y mediante un extrusor se deposita por capas hasta formar un sólido en tres dimensiones. Las características del material, la temperatura de fundido, la distribución de capas, entre otros parámetros, influirán no solo en el aspecto y acabado de la pieza impresa, sino también en su resistencia. Debe transmitirse al alumno la necesidad de que las piezas impresas en $3 \mathrm{D}$ cumplan unos requerimientos que cualquier componente industrial debe verificar. 
Dado que la práctica aquí sugerida tiene la intención de aplicarse a distintos niveles universitarios de grado y posgrado, la complejidad de los ensayos y sus implicaciones también tienen una gran amplitud. El ensayo más sencillo y paradigma de la resistencia de materiales es el ensayo de tracción. Dicho ensayo es imprescindible en la caracterización de materiales y además sirve para tratar conceptos básicos en la Mecánica de Medios Continuos:

- Tensiones y deformaciones.

- Elasticidad y efecto Poisson.

- Anisotropía.

- Fluencia y estricción.

- Rotura y ductilidad.
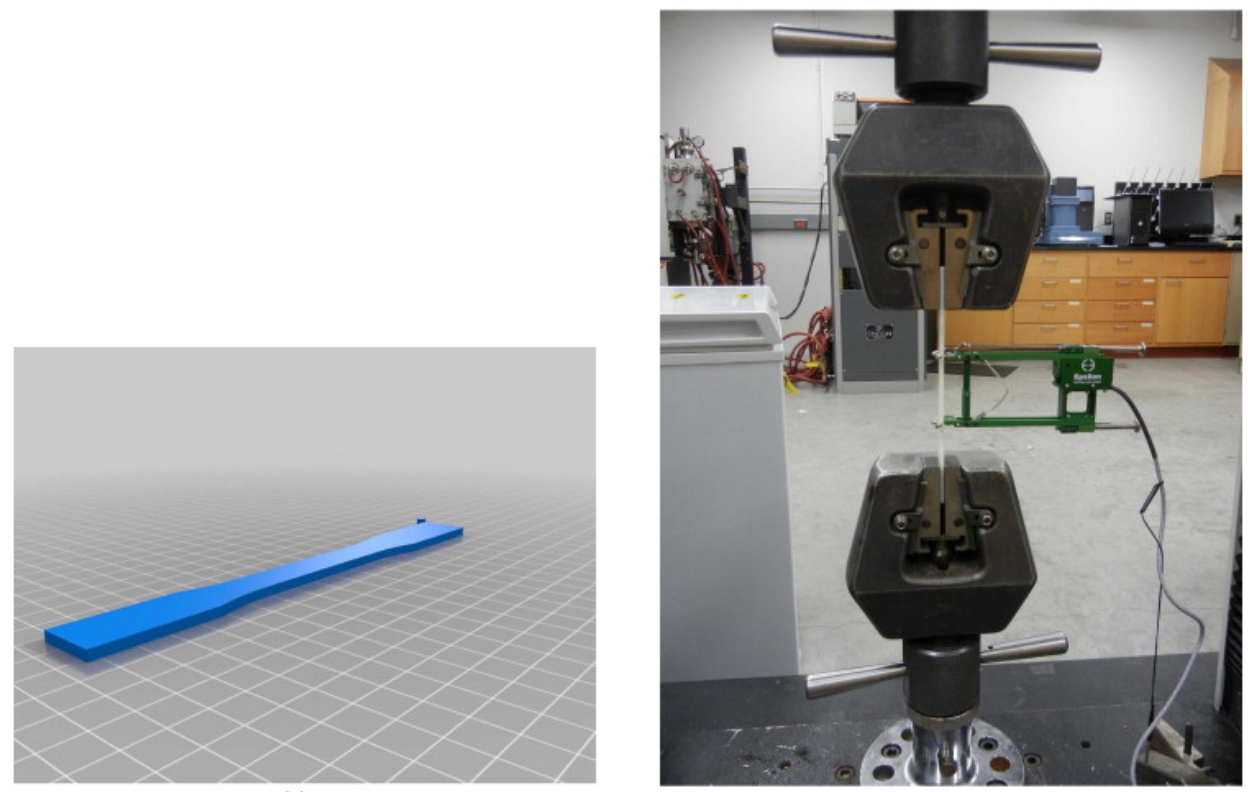

Fig. 1: Diseño de probeta de tracción y máquina de ensayo (extraído de Tymrak, Kreiger y Pearce 2014)

Este ensayo está normalizado para la caracterización de materiales, en concreto la norma UNE-EN ISO 527-1:2012 describe el procedimiento para plásticos. Por lo tanto, la combinación de la Impresión 3D con la realización de ensayos de tracción es una práctica factible en asignaturas de resistencia y ciencia de materiales durante los primeros cursos. Sin embargo, en cursos avanzados o niveles de posgrado suelen requerirse ensayos más complejos relacionados con la Integridad Estructural:

- Ensayos de Fractura. Aquellos ensayos en los que las probetas tienen un concentrador de tensiones, es decir, una entalla. Los fundamentos de la Mecánica de la Fractura pueden explicarse en laboratorio mediante este tipo de ensayos 
cuyo objetivo es la predicción y prevención de fallos catastróficos en estructuras y componentes industriales. Se ahondará en los conceptos de tenacidad y comportamiento dúctil frente a frágil.

- Ensayos de fluencia a alta temperatura. El fenómeno conocido como Creep es muy importante en la resistencia de componentes sometidos a altas temperaturas, especialmente en los polímeros.

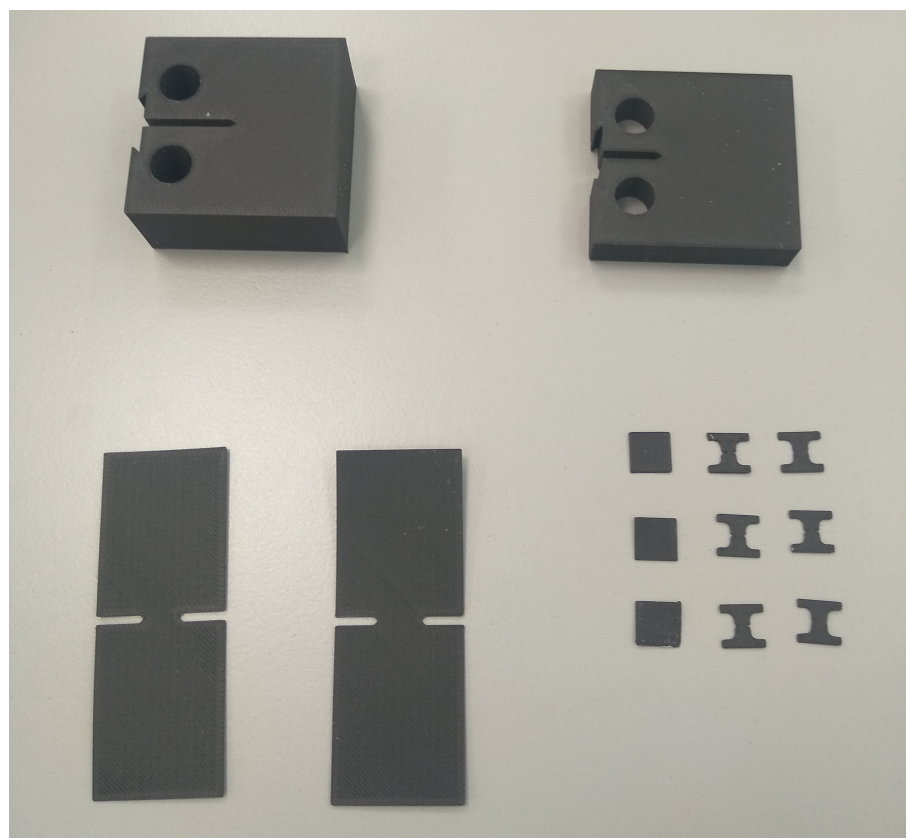

Fig. 2: Diferentes tipos de probetas impresas en 3D

\section{Conclusiones}

El presente trabajo ha tratado de revisar el papel de la Impresión 3D en la docencia. Por un lado, se trata de una tecnología novedosa con la que tanto el alumnado como el profesorado deben familiarizarse, especialmente en carreras técnicas y relacionadas con la industria donde la Fabricación Aditiva es un sector cada vez más competitivo y que requerirá un gran número de profesionales cualificados en los próximos años. En cuanto a su utilidad como herramienta docente, además del valor intrínseco desde un punto de vista tecnológico, la Impresión 3D permite la producción de objetos de representación que facilitan un aprendizaje tangible mediante modelos de realidad. La visualización en tres dimensiones puede ser clave para la asimilación de determinados conceptos aunque no es condición suficiente y debe combinarse con otras estrategias de aprendizaje más abstracto o simbólico. A pesar de que el presente trabajo puede definirse como una propuesta teórica dentro de un debate sobre funcionalidad didáctica de la tecnología de Impresión 3D, no debe olvidarse el horizonte práctico a corto plazo y su implementación futura en guías docentes dentro de determinadas asignaturas. 
Se destaca de forma particular la posible introducción de esta metodología en materias de Resistencia de Materiales o de Integridad Estructural ya que en estos casos la caracterización de materiales y de sus propiedades mecánicas se facilita de manera enorme mediante la técnica de la Impresión 3D. De este modo, se propone un escenario docente que abarque desde la fase de concepción de una pieza hasta su ensayo en laboratorio. Este enfoque integral tiene obvias limitaciones de tiempo, recursos o diseño curricular. Sin embargo, el nivel de detalle en cada una de las fases variará dependiendo de los objetivos de cada asignatura. En aquellos casos en los que el objetivo principal sea la asimilación de la tecnología de Impresión 3D, el ensayo de piezas será accesorio mientras que cuando los conceptos de resistencia sean fundamentales, dicha técnica será solamente una herramienta de apoyo.

\section{Referencias bibliográficas}

De Jong, Jeroen PJ y Erik de Bruijn (2013). "Innovation lessons from 3-D printing". En: MIT Sloan Management Review 54.2, pág. 43.

Dunleavy, Matt y Chris Dede (2014). "Augmented reality teaching and learning". En: Handbook of research on educational communications and technology. Springer, págs. 735-745.

Ford, Simon y Tim Minshall (2018). "Invited Review Article: Where and how 3D printing is used in teaching and education". En: Additive Manufacturing.

Gentner, Dedre y Keith J Holyoak (1997). "Reasoning and learning by analogy: Introduction." En: American psychologist 52.1, pág. 32.

Huk, Thomas (2006). "Who benefits from learning with 3D models? The case of spatial ability". En: Journal of computer assisted learning 22.6, págs. 392-404.

Huleihil, M (2017). "3D printing technology as innovative tool for math and geometry teaching applications". En: IOP Conference Series: Materials Science and Engineering. Vol. 164. 1. IOP Publishing, pág. 012023.

Katsioloudis, Petros y Millie Jones (2015). "Using computer-aided design software and 3D printers to improve spatial visualization". En: Technology and Engineering Teacher 74.8, pág. 14 .

Kietzmann, Jan, Leyland Pitt y Pierre Berthon (2015). "Disruptions, decisions, and destinations: Enter the age of 3-D printing and additive manufacturing". En: Business Horizons 58.2, págs. 209-215.

Kong, Xiangxue y col. (2016). "Do Three-dimensional Visualization and Three-dimensional Printing Improve Hepatic Segment Anatomy Teaching? A Randomized Controlled Study". En: Journal of Surgical Education 73.2, págs. 264-269. ISSN: 19317204. DOI: https://doi.org/10.1016/j.jsurg.2015.10.002.

Lacey, Gary (2010). "3D printing brings designs to life". En: Tech Directions 70.2, pág. 17. 
Lateef, Fatimah (2010). "Simulation-based learning: Just like the real thing". En: Journal of Emergencies, Trauma and Shock 3.4, pág. 348.

Marshall, Paul, Sara Price e Yvonne Rogers (2003). "Conceptualising Tangibles to Support Learning". En: Proceedings of the 2003 Conference on Interaction Design and Children. IDC '03. Preston, England: ACM, págs. 101-109. ISBN: 1-58113732-X. DOI: $10.1145 / 953536.953551$.

Martin, Robert L, Nicholas S Bowden y Chris Merrill (2014). "3D printing in technology and engineering education". En: Technology and engineering teacher 73.8, pág. 30 .

Meij, Jan van der y Ton de Jong (2006). "Supporting students' learning with multiple representations in a dynamic simulation-based learning environment". En: Learning and instruction 16.3, págs. 199-212.

Pérez, José Luis Saorın y col. (2015). "Blokify: Juego de modelado e impresión 3D en tableta digital para el aprendizaje de vistas normalizadas y perspectiva". En: Digital Education Review 27, págs. 105-121.

Rodenbough, Philip P, William B Vanti y Siu-Wai Chan (2015). "3D-printing crystallographic unit cells for learning materials science and engineering". En: Journal of Chemical Education 92.11, págs. 1960-1962.

Tymrak, BM, M Kreiger y Joshua M Pearce (2014). "Mechanical properties of components fabricated with open-source 3-D printers under realistic environmental conditions". En: Materials \& Design 58, págs. 242-246.

Vaccarezza, Mauro y Veronica Papa (2015). "3D printing: a valuable resource in human anatomy education". En: Anatomical Science International 90.1, págs. 64-65. ISSN: 1447-073X. DOI: 10.1007/s12565-014-0257-7.

Villegas, Gonzalo Ulloa (2011). "Rethinking Engineering Education. The CDIO approach. Edward Crawley, Johan Malmqvist, Sören Östlund y Doris Brodeur. Springer (New York, 2007). ISBN: 978-0-387-38287-6. Cubierta dura, 286 páginas". En: Sistemas \& Telemática 9.16, págs. 91-92.

Winn, William (1982). "Visualization in learning and instruction: a cognitive approach". En: ECTJ 30.1, págs. 3-25. ISSN: 1556-6501. DOI: 10.1007/BF02766544. 